\title{
Neurological functions of the masterswitch protein kinase - GSK-3
}

\author{
Oksana Kaidanovich-Beilin ${ }^{1}$, Jean-Martin Beaulieu ${ }^{2}$, Richard Scott Jope ${ }^{3}$ and James Robert Woodgett ${ }^{1,4 *}$ \\ ' Samuel Lunenfeld Research Institute, Mount Sinai Hospital, Toronto, ON, Canada \\ 2 Department of Psychiatry and Neuroscience, Institut Universitaire de Santé Mentale de Québec, Université Laval, Québec City, QC, Canada \\ ${ }^{3}$ Department of Psychiatry and Behavioral Sciences, University of Miami, Miami, FL, USA \\ ${ }^{4}$ Department of Medical Biophysics, University of Toronto, Toronto, ON, Canada \\ *Correspondence: beilin@/unenfeld.ca; woodgett@/unenfeld.ca
}

I know that I know nothing (Socrates, 469 BC-399 BC)

This Research Topic is dedicated to a rather unique cellular regulator termed Glycogen Synthase Kinase-3 (GSK-3). In the late 1970s, this protein serine kinase was first discovered through its ability to activate the ATP-Mg-dependent form of type-1 protein phosphatase (Factor A activity) and to phosphorylate the key ratelimiting metabolic enzyme that catalyzes the last step of glycogen synthesis, glycogen synthase. From five chromatographically separable glycogen synthase kinases, the third (GSK-3) was subsequently demonstrated to mediate the effect of insulin on activation of glycogen synthesis: insulin leads to inactivation of GSK-3 which relieves its inhibitory phosphorylation of glycogen synthase. Molecular cloning in 1990 revealed the existence of two distinct genes for GSK-3, which encode two paralogous signaling proteins termed GSK-3 $\alpha$ and GSK-3 $\beta$ (reviewed in Kaidanovich-Beilin and Woodgett, 2011). GSK-3 has intrigued many researchers for over 30 years by its unusual features, mechanisms of regulation, its participation within at least four major signaling systems, and its capacity to influence the functions of more than 100 substrates (reviewed in Kaidanovich-Beilin and Woodgett, 2011).

While GSK-3 was initially identified as a regulator of metabolism, a significant role in neuroscience has since emerged. The first indication of an important brain function was made in 1992 by Hanger et al. (1992) who showed that GSK-3 phosphorylates the microtubule-associated protein tau at sites relevant to the pathology of Alzheimer's disease, further supported by studies by Takahashi et al. (1994) who demonstrated that Tau was indeed a physiological substrate of GSK-3, which at that time was also called tau protein kinase I (TPKI) (reviewed in Kremer et al., 2011; Medina et al., 2011; Takashima, 2012). These findings were important milestones for initiating interest in GSK-3 in the pathogenesis of neurodegenerative disorders, such as Alzheimer's disease (see Gomez-Sintes et al., 2011; Kremer et al., 2011; Medina et al., 2011; Takashima, 2012).

In parallel to the aforementioned discoveries, GSK-3 was shown to be a major regulator of the Wnt signaling pathway. In 1995, studies in Xenopus laevis showed that the expression of a catalytically inactive mutant of GSK-3 induces duplication of the dorsal axis, a phenotype similar to earlier studies of lithium ion treatment of Xenopus embryos. A molecular explanation for this relationship was discovered in 1996 when GSK-3 was shown to be inhibited by lithium (see chapter by Valvezan and Klein, 2012). Lithium was a gold standard in the treatment of bipolar disorder (reviewed by Freland and Beaulieu, 2012). This finding poured gas onto the GSK-3 fire in neuroscience research. Subsequent multiple studies have shown associations between dysregulation of GSK3-mediated signaling pathways and the pathogenesis of bipolar disorder (reviewed in Jope, 2011), Fragile X syndrome (reviewed in Mines and Jope, 2011), schizophrenia (reviewed in Emamian, 2012), brain tumors (reviewed in Mills et al., 2011), and stroke (reviewed in Chuang et al., 2011). Several independent studies have also shown the involvement of GSK-3 in the action of the monoamine neurotransmitters serotonin and dopamine that are central targets for antidepressant and antipsychotic drugs (reviewed in Beaulieu et al., 2011; Polter and Li, 2011) therefore suggesting that GSK-3 may constitute an important "missing link" between disease mechanisms and treatments for mental illnesses.

In 2000, the first mouse knockout model of GSK-3 (beta) was reported (reviewed in Kaidanovich-Beilin and Woodgett, 2011), and provided the first of many tools for physiological analysis of GSK-3's roles. Subsequent transgenic models of GSK-3 have allowed its role to be elucidated in the mechanism of monoamine neurotransmitter action (reviewed in Beaulieu et al., 2011; Polter and $\mathrm{Li}, 2011$ ) and synaptic plasticity (reviewed in Bradley et al., 2012), neurodevelopment (reviewed in Kim and Snider, 2011; Cole, 2012), neuronal morphogenesis (reviewed in Kim et al., 2011), apoptosis, and neuronal survival (reviewed in Gomez-Sintes et al., 2011) as well as neuroinflammation (reviewed in Beurel, 2011).

Hence a single pair of signaling molecules can be found at the center of multiple neuronal functions. Indeed, these roles positioned GSK-3 as a potential therapeutic target for both psychiatric and neurodegenerative diseases and a series of pharmacological inhibitors with alternative modes of actions have been generated over last two decades (reviewed in Eldar-Finkelman and Martinez, 2011). We certainly hope that these molecules will prove useful and safe in the clinic as there are many unmet needs in both psychiatry and dementia-related medicine. That said, study of GSK-3 in the brain has uncovered many important facets of brain biology and there is clearly much more to be revealed.

\section{ACKNOWLEDGMENTS}

We thank all of the contributors to this volume from around the world (UK, Belgium, Italy, Spain, Israel, Japan, Australia, USA, and Canada) for their informative reviews of the many different facets of GSK-3 neurobiology. We also appreciate the effort of the reviewers for their comprehensive manuscript evaluations and suggestions. 


\section{REFERENCES}

Beaulieu, J. M., Del'guidice, T., Sotnikova, T. D., Lemasson, M., and Gainetdinov, R. R. (2011). Beyond cAMP: the regulation of Akt and GSK3 by dopamine receptors. Front. Mol. Neurosci. 4:38. doi: 10.3389/ fnmol.2011.00038

Beurel, E. (2011). Regulation by glycogen synthase kinase- 3 of inflammation and $\mathrm{T}$ cells in CNS diseases. Front. Mol. Neurosci. 4:18. doi: 10.3389/ fnmol.2011.00018

Bradley, C. A., Peineau, S., Taghibiglou, C., Nicolas, C. S., Whitcomb, D. J., Bortolotto, Z. A., Kaang, B. K., Cho, K., Wang, Y. T., and Collingridge, G. L. (2012). A pivotal role of GSK-3 in synaptic plasticity. Front. Mol. Neurosci. 5:13. doi: 10.3389/ fnmol.2012.00013

Chuang, D. M., Wang, Z., and Chiu, C. T. (2011). GSK-3 as a target for lithium-induced neuroprotection against excitotoxicity in neuronal cultures and animal models of ischemic stroke. Front. Mol. Neurosci. 4:15. doi: 10.3389/fnmol.2011.00015

Cole, A. R. (2012). GSK3 as a sensor determining cell fate in the brain. Front. Mol. Neurosci. 5:4.doi: 10.3389/ fnmol.2012.00004

Eldar-Finkelman, H., and Martinez, A. (2011). GSK-3 inhibitors: preclinical and clinical focus on CNS. Front.
Mol. Neurosci. 4:32. doi: 10.3389/ fnmol.2011.00032

Emamian,E.S. (2012). AKT/GSK3 signaling pathway and schizophrenia. Front. Mol. Neurosci. 5:33. doi: 10.3389/ fnmol.2012.00033

Freland, L., and Beaulieu, J. M. (2012). Inhibition of GSK3 by lithium, from single molecules to signaling networks. Front. Mol. Neurosci. 5:14. doi: 10.3389/fnmol.2012.00014

Gomez-Sintes, R., Hernandez, F., Lucas, J. J., and Avila, J. (2011). GSK-3 mouse models to study neuronal apoptosis and neurodegeneration. Front. Mol. Neurosci. 4:45. doi: 10.3389/ fnmol.2011.00045

Hanger, D. P., Hughes, K., Woodgett, J. R., Brion, J. P., and Anderton, B. H. (1992). Glycogen synthase kinase-3 induces Alzheimer's disease-like phosphorylation of Tau: generation of paired helical filament epitopes and neuronal localisation of the kinase. Neurosci. Lett. 147, 58-62.

Jope, R. S. (2011). Glycogen synthase kinase- 3 in the etiology and treatment of mood disorders. Front. Mol. Neurosci. 4:16. doi: 10.3389/ fnmol.2011.00016

Kaidanovich-Beilin, O., and Woodgett, J. R. (2011). GSK-3: functional insights from cell biology and animal models. Front. Mol. Neurosci. 4:40. doi: 10.3389/fnmol.2011.00040
Kim, W. Y., and Snider, W. D. (2011). Functions of GSK-3 signaling in development of the nervous system. Front. Mol. Neurosci. 4:44. doi: 10.3389/ fnmol.2011.00044

Kim, Y. T., Hur, E. M., Snider, W. D., and Zhou, F. Q. (2011). Role of GSK3 signaling in neuronal morphogenesis. Front. Mol. Neurosci. 4:48. doi: 10.3389/fnmol.2011.00048

Kremer, A., Louis, J. V., Jaworski, T., and Van Leuven, F. (2011). GSK3 and Alzheimer's disease: facts and fiction. Front. Mol. Neurosci. 4:17. doi: 10.3389/fnmol.2011.00017

Medina, M., Garrido, J. J., and Wandosell, F. G. (2011). Modulation of GSK-3 as a therapeutic strategy on Tau pathologies. Front. Mol. Neurosci. 4:24. doi: 10.3389/fnmol.2011.00024

Mills, C. N., Nowsheen, S., Bonner, J. A., and Yang, E. S. (2011). Emerging roles of glycogen synthase kinase 3 in the treatment of brain tumors. Front. Mol. Neurosci. 4:47. doi: 10.3389/ fnmol.2011.00047

Mines, M. A., and Jope, R. S. (2011). Glycogen synthase kinase-3: a promising therapeutic target for Fragile X syndrome. Front. Mol. Neurosci. 4:35. doi: 10.3389/fnmol.2011.00035

Polter, A. M., and Li, X. (2011) Glycogen synthase kinase- 3 is an intermediate modulator of serotonin neurotransmission. Front.
Mol. Neurosci. 4:31. doi: 10.3389/ fnmol.2011.00031

Takahashi, M., Tomizawa, K., Kato, R., Sato, K., Uchida, T., Fujita, S. C., and Imahori, K. (1994). Localization and developmental changes of tau protein kinase I/glycogen synthase kinase-3 beta in rat brain. J. Neurochem. 63, 245-255.

Takashima, A. (2012). GSK-3 and memory formation. Front. Mol. Neurosci. 5:47. doi: 10.3389/fnmol.2012.00047

Valvezan, A. J., and Klein, P. S. (2012). GSK-3 and Wnt signaling in neurogenesis and bipolar disorder. Front. Mol. Neurosci. 5:1. doi: 10.3389/ fnmol.2012.00001

Received: 08 February 2012; accepted: 23 March 2012; published online: 09 April 2012.

Citation: Kaidanovich-Beilin O, Beaulieu $J-M$, Jope RS and Woodgett JR (2012) Neurological functions of the masterswitch protein kinase - GSK-3. Front. Mol. Neurosci. 5:48. doi: 10.3389/ fnmol.2012.00048

Copyright (c) 2012 Kaidanovich-Beilin, Beaulieu, Jope and Woodgett. This is an open-access article distributed under the terms of the Creative Commons Attribution Non Commercial License, which permits non-commercial use, distribution, and reproduction in other forums, provided the original authors and source are credited. 